\title{
An Empirical Study on the Benefit of Split Loads with the Pickup and Delivery Problem
}

\author{
Maciek Nowak \\ Loyola University Chicago, mnowak4@luc.edu \\ Chelsea C. White \\ Georgia Institute of Technology - Main Campus \\ Ozlem Ergun \\ Georgia Institute of Technology - Main Campus
}

Follow this and additional works at: https://ecommons.luc.edu/isom_facpubs

Part of the Business Administration, Management, and Operations Commons, Management Information Systems Commons, and the Technology and Innovation Commons

Author Manuscript

This is a pre-publication author manuscript of the final, published article.

\section{Recommended Citation}

Nowak, Maciek; White, Chelsea C.; and Ergun, Ozlem. An Empirical Study on the Benefit of Split Loads with the Pickup and Delivery Problem. European Journal of Operational Research, 198, 1: , 2009. Retrieved from Loyola eCommons, Information Systems and Operations Management: Faculty Publications \& Other Works, http://dx.doi.org/10.1016/j.ejor.2008.09.041

This Article is brought to you for free and open access by the Faculty Publications and Other Works by Department at Loyola eCommons. It has been accepted for inclusion in Information Systems and Operations Management: Faculty Publications \& Other Works by an authorized administrator of Loyola eCommons. For more information, please contact ecommons@luc.edu. c) $(1) \ominus$

This work is licensed under a Creative Commons Attribution-Noncommercial-No Derivative Works 3.0 License. (C) 2008 Elsevier B.V. 


\title{
An Empirical Study on the Benefit of Split Loads with the Pickup and Delivery Problem
}

\author{
Maciek Nowak $^{\mathrm{a}, *}$,Ozlem Ergun ${ }^{\mathrm{b}}$, Chelsea C. White, III ${ }^{\mathrm{b}}$ \\ ${ }^{a}$ Loyola University Chicago, Department of Information Systems and Operations \\ Management, 1 E. Pearson Ave., Chicago, IL, 60611, USA, voice: 312-915-6113, \\ fax: 312-915-7207 \\ ${ }^{\mathrm{b}}$ Georgia Institute of Technology, School of Industrial and Systems Engineering, \\ 765 Ferst Drive, NW, Atlanta, Georgia, 30332, USA
}

\begin{abstract}
Splitting loads such that the delivery of certain loads is completed in multiple trips rather than one trip has been shown to have benefit for both the classic Vehicle Routing Problem and the Pickup and Delivery Problem. However, the magnitude of the benefit may be affected by various problem characteristics. In this paper, we characterize those real world environments in which split loads are most likely to be beneficial. Based on practitioner interest, we determine how the benefit is affected by the mean load size and variance, number of origins relative to the number of destinations, the percentage of origin-destination pairs with a load requiring service, and the clustering of origin and destination locations. We find that the magnitude of benefit: is greatest for load sizes just over one half vehicle capacity as these loads can not be combined without splitting, while they are the easiest to combine on a vehicle with splitting; increases as the number of loads sharing an origin or destination increases because there are more potential load combinations to split at each stop; and increases as the average distance from an origin to a destination
\end{abstract}


increases because splitting loads reduces the trips from origins to destinations.

Key words: transportation, vehicle routing, split pickup and delivery

\section{$1 \quad 1$ Introduction}

2 Splitting loads such that the delivery of certain loads is completed in multi-

3 ple trips rather than one trip results in opportunities for a reduction in cost

4 and the number of vehicles used. Several studies have shown the benefit of

5 split deliveries for the classic Vehicle Routing Problem (VRP), in which a

6 vehicle operating out of a depot makes a series of deliveries on each route

7 ((Dror et al., 1994), (Frizzell and Giffin, 1995), (Sierksma and Tijssen, 1998),

8 (Archetti et al., 2006)). More recently, Nowak et al. (2008) quantified the ben-

9 efit for the Pickup and Delivery Problem (PDP), in which a vehicle picks up

10 a load from a specific origin and delivers it to its destination. They showed

11 theoretically that the optimal load size for splitting is just above one half of

12 a truckload and supported this result with empirical evidence. Furthermore,

13 a real world example was used to show that certain problem characteristics

14 may limit the benefit of split loads.

15 Although the theoretical results are of interest, practitioners have found the

16 results regarding the characteristics of the problem that have an effect on the

17 benefit of split loads to be of more use. The real world case presented in Nowak

18 et al. (2008) showed that these benefits are affected by the per stop cost asso-

* Corresponding author.

Email addresses: mnowak4@luc.edu (Maciek Nowak), oergun@isye.gatech.edu

(Ozlem Ergun), cwhite@isye.gatech.edu (Chelsea C. White, III). 
ciated with each pickup or delivery, the size of the loads requiring service and

the number of common origins or destinations requiring service. In this paper, we determine the degree to which these characteristics impact the benefit. We focus on the latter two characteristics of real world environments, the size of loads to be delivered and the distribution of flow over the network, while also analyzing the geographic orientation of origins and destinations. Specifically, we determine how the magnitude of benefit is affected by mean load size and variance, the number of origins relative to the number of destinations, the percentage of origin-destination pairs with a load requiring service, and the clustering of origin and destination locations.

We find that the magnitude of benefit: is greatest for load sizes just over one half vehicle capacity as these loads can not be combined without splitting, while they are the easiest to combine on a vehicle with splitting; increases as the number of loads sharing an origin or destination increases because there are more potential load combinations to split at each stop; and increases as the average distance from an origin to a destination increases because splitting loads reduces the trips from origins to destinations. Through this analysis, practitioners will find a guide describing those instances where splitting loads is most beneficial, as well as instances where the additional effort associated with load splitting is not justified.

This paper is organized as follows. Section 2 describes the experimental design including the common traits shared by all problem instances tested. Section 3 presents the results from the tests on the mean load size and variance. Section 4 describes the effect that the number of origins relative to the number of destinations has on the magnitude of benefit from split loads. Section 5 4 discusses how benefit is affected by the number of loads to be serviced from 
a common origin or to a common destination. Section 6 analyzes two types

46 of location clusters and how they influence the benefit. Section 7 summarizes

${ }_{47}$ the results of the paper.

\section{${ }_{48} \quad 2 \quad$ Experimental Design}

49

Several sets of problem instances are generated using the different characteristics to be tested, as described in the following sections. However, all of the instances share several common traits. The majority of problem sets tested have 50, 100 or 150 transportation requests, as these sizes are similar to the problem sizes used in testing of the SDVRP (Dror and Trudeau, 1989, 1990). Each transportation request contains the origin and destination location coordinates and the fraction of a truckload to be delivered. X and Y coordinates for the pickup and delivery locations and load sizes are randomly generated. The locations are uniformly distributed over the range $[-40,40]$ for both $\mathrm{X}$ and Y coordinates for the problems in Sections 3-5, while Section 6 analyzes different distributions for the locations. The load sizes are all less than or equal to vehicle capacity, which is set at one, without loss of generality. This is done to determine the load sizes that benefit most from splitting that can otherwise be serviced by a vehicle in one trip without splitting. The case study discussed by Nowak et al. (2008) presented tests run with load sizes greater than vehicle capacity.

The heuristic developed in Nowak et al. (2008) is used to solve each problem under two scenarios, both with and without split loads. This heuristic functions by randomly generating a split load for a solution that initially has a unique route dedicated to each load. After the split load is created, local search 
techniques, such as route combination, load swapping and insertion, are used to improve the solution. Additional splits are created and local improvements made until no cost reduction is found. The use of a heuristic is justified as both the PDP and PDPSL are NP-hard (Nowak et al., 2008). The cost of the solution in each case is equivalent to the distance traveled by the vehicle. The two costs are compared to determine the percentage cost reduction that is found through the use of split loads. An additional constraint is implemented limiting routes to 500 miles. While loads could still be combined on a vehicle due to the relative proximity of stops, this prevents a vehicle from servicing all of the loads on one route.

In order to evaluate the heuristic, it was tested on a set of eleven standard TSPLIB problem instances, finding solutions within $5 \%$ of the best known cost of seven instances and within $10 \%$ of all instances. Given that the heuristic was designed to focus on the additional complexities associated with the PDP, we find these results to be acceptable. The heuristic was coded in $\mathrm{C}$ and all experiments were run on a 2.4-GHz Xeon processor with a 400-Mhz frontside bus and 2 GB RAM.

\section{Mean Load Size and Variance}

Previous research has shown that the most benefit from split loads occurs with load sizes just over one half vehicle capacity. The following theorem was presented by Nowak et al. (2008):

Theorem 1 Given the origin and destination locations of a set of $k$ loads, a vehicle of capacity $Q$, and a very small value, $\epsilon$, let $v(P D P S L)$ be the cost of the optimal PDPSL solution to deliver these loads and $v(P D P)$ be the cost of 
the optimal PDP solution. Then the ratio $v(P D P) / v(P D P S L)$ is maximized when the loads are all of size $Q / 2+\epsilon$, as $k \rightarrow \infty$.

This theorem was supported with tests run on a variety of load instances uniformly generated over several load size ranges. While these results provide some basic insight into those loads that are most likely to lead to a cost reduction through splitting, a more in depth look at mean load size and variance is of interest. Classifying industries by the size of loads delivered is difficult for most goods transported, so this analysis will not define those sectors that are most likely to gain benefit from split loads based on load size. However, the following tests may provide a simple guide for any company wishing to determine if split loads should be considered based on the load sizes they generally transport. Archetti et al. (2008) studied the effect of mean load size and variance on split loads for the VRP, finding results similar to those reported here. Prior to an analysis of load size variance, the benefit for various load sizes with no variance is presented with a more defined picture than that found in Nowak et al. (2008). Determining the benefit without variance provides a baseline indicating the exact load sizes for which the most and least benefit may be found over the range from zero to one truckload.

Problem instances with 50, 100 and 150 load requests of a common size are generated with load sizes incremented by 0.05 truckloads (TL) in the range [0.05 - 0.95]. Additional problems are tested for those sizes where significant changes in benefit may be expected. These load sizes include $0.11,0.21,0.26$, 0.33, 0.34 and 0.51 . Three different sets of location coordinates are randomly generated to test each load size. Figure 1 displays the reduction in cost with split loads for each of the load sizes tested, where the results are very similar for each number of load requests. Almost all benefit from the use of split loads 
is eliminated for the sizes $0.1,0.2,0.25,0.33$, and 0.5 , or $Q / k$ for $k=2, \ldots$, where $Q=1$ is vehicle capacity. These load sizes can easily be combined on a vehicle with no splitting required. Peaks in cost reduction are found for the load sizes $0.11,0.21,0.26,0.34$, and 0.51 . When splitting is allowed, these load sizes may have as little as 0.01 TL split off to allow for loads to be placed on a vehicle simultaneously. Although these results show the load sizes that provide the most (and least) benefit with split loads, it is rare to find a set of circumstances in the real world where all loads to be transported are of a common size. Therefore, it is of interest to determine the effect that load size variance has on this benefit.

Fig. 1. Average Percentage Cost Reduction with Split Loads for Load Sizes in the Range $[0.05-0.95]$

New problem instances are randomly generated with means ranging from 0.05 to $0.95 \mathrm{TL}$ and with variances from 0.005 to 0.08 . A beta distribution is used to generate the load sizes as this distribution is defined on the interval $[0,1]$ and all loads for the problems described here are of a size less than or equal to one truckload. This distribution is parameterized by two non-negative shape parameters, typically denoted by $\alpha$ and $\beta$, which are estimated using the method of moments with the following two equations:

$$
\begin{aligned}
& \alpha=x\left(\frac{x(1-x)}{v}-1\right), \\
& \beta=(1-x)\left(\frac{x(1-x)}{v}-1\right),
\end{aligned}
$$


the GNU Scientific Library. Two different problem instances are randomly generated with load sizes corresponding to each mean and variance. Two sets of location coordinates are also randomly generated, such that four problem instances are tested for each mean and variance.

Figure 2 provides the cost reduction for each variance, overlayed by the results displayed in Figure 1 for which there is no variance. Any variance has an immediate effect on the benefit of split loads. For those load size means below one half of a truckload the peaks and minima are virtually eliminated. For the variance of 0.005 there are two slight dips, one at $0.45 \mathrm{TL}$ and the other at 0.2 - 0.25 TL. The variation is small enough such that the majority of load sizes are still in the range [0.4-0.5] for the mean of 0.45 and [0.15-0.3] for the mean of 0.2 or 0.25 . Loads in these ranges are easily combined on a vehicle with no splitting required. The other variances tested display an almost constant percentage of cost reduction for each mean up to 0.5 TL. Load size means just above one half of a truckload still result in a greater cost reduction, even with a variance as high as 0.04 . However, the peak in cost reduction diminishes as the variance increases. A greater variance for a mean load size above one half vehicle capacity results in problem sets with more load sizes below one half vehicle capacity, allowing for more loads to be combined on a vehicle without splitting and a reduction in benefit. Similarly, a greater variance for a mean load size below one half vehicle capacity results in problem sets with more load sizes above one half vehicle capacity and more splitting required to combine loads on a vehicle, with an increase in benefit. This is further illustrated in the following table.

Table 1 presents the average cost reduction over all load size means for each variance. Although the problem instances with no variance result in the widest 
Fig. 2. Average Percentage Cost Reduction with Split Loads for each Load Size Mean and Variance

166 are most visible as other problem characteristics are altered.

Table 1

Average Percentage Cost Reduction with Split Loads for the Tested Load Size Variances

range of values for cost reduction, the average reduction is not significantly greater than those instances with some variance. Most real world problems will have varying load sizes and these results indicate that there is some benefit associated with almost any mean and variance combination. However, there is a clear difference between loads with a mean size above and below one half a truckload. Table 1 also separates the average percentage cost reduction to show this distinction. As is evident in Figure 2, there is a greater benefit for load sizes with a mean greater than half a truckload at variances up to 0.04 .

Generalizing these results for any real world case is difficult, as most industries can not be classified by the load sizes in which their goods are transported. However, this is a very important factor in determining if splitting loads will provide a significant benefit. When the majority of load sizes are clustered around one half of a truckload, split loads should provide an opportunity for cost savings. Other load sizes may result in a benefit, but it would most likely be reduced. The load sizes used for the remaining problem instances in this study fall in the range $[0.51-0.60]$, as loads of this size result in the most opportunity for benefit from split loads. Because of this, changes in benefit 


\section{Number of Origins and Destinations}

When the Pickup and Delivery Problem has only one origin or one destination it is reduced to the Vehicle Routing Problem. As described earlier, the benefits associated with using split loads with the VRP have been quantified. Relaxing the VRP to allow for multiple origins and destinations raises the question of how that benefit is affected by the ratio of the number of origins to the number of destinations. This should allow for a comparison between industries with heavy inbound or outbound flow and those with a mixed flow. Industries with heavy inbound flow, where a large number of materials or parts are used to make few products (ie, auto industry), should have loads leaving from many origins with a few common destinations, while heavy outbound flow, where few materials make many products (ie, chemical industry), should be characterized by loads leaving from a few common origins to many destinations.

To determine the effect of the number of origins relative to number of destinations on the magnitude of benefit, problem instances with various ratios are tested. To minimize variability between problems the ratios are selected such that each problem requires the delivery of a similar number of loads, 50, 100 or 150. The ratios of the seven 50 load problem sets tested are: (number of origins : number of destinations) $25: 2,12: 4,10: 5,7: 7,5: 10,4: 12$, and $2: 25$. The ratios of the nine 100 load problem sets tested are: $50: 2,25: 4,20: 5,14$ : $7,10: 10,7: 14,5: 20,4: 25$, and $2: 50$. The ratios of the nine 150 load problem sets tested are: $75: 2,37: 4,30: 5,15: 10,12: 12,10: 15,5: 30,4: 37$, and $2: 75$. Six different sets of location coordinates and five different sets of load sizes are randomly generated for each ratio, resulting in 30 problem instances for each number of loads. 


$$
\text { or destination is not as great. }
$$

Fig. 3. Average Percentage Cost Reduction with Split Loads for Problem Instances with a Varying Number of Origins and Destinations

Figure 3 presents the cost reduction for each of the ratios. All ratios result in a cost reduction between 25 and $34 \%$. The most benefit is found in the instances that most closely represent the VRP, with either two origins or two destinations. Benefit is reduced as the ratio of the number of origins to the number of destinations approaches one. This is because opportunities for load splitting grow as the number of loads departing from or arriving to a common location increases. In the instance with two origins and 150 loads, when the vehicle arrives for a pick up there are 75 different loads to select from to create a combination of split loads. Dropping off loads at only two destinations has similar opportunities. The instances with ratios of $37: 4$ and $4: 37$ have 38 fewer loads leaving from or arriving to any origin or destination, thereby resulting in the largest decline in cost reduction. Less variance is found between the other ratios as the change in the number of loads available at each origin

These results indicate that split loads would be most beneficial in a situation where many loads are departing from or arriving to a common location. As with the industry example described earlier, this indicates that the most benefit would be found in the supply chain for production processes with heavy inbound flow or heavy outbound flow. These supply chains have many loads sharing common origins or destinations that provide for the most potential split load combinations. For the remainder of this paper we will report results for the ratios $5: 10,5: 20,10: 10$ and $10: 15$. The results for other ratios are similar, with overall cost reduction slightly increased or decreased dependent 
on the ratio.

\section{Origin-Destination Pairs Requiring Service}

Every origin-destination pair has a load requiring service in each problem instance generated above. However, as shown by Nowak et al. (2008), a real world problem instance will likely not have this characteristic and this may have an effect on the benefit of split loads. To evaluate this effect, the percentage of origin-destination pairs requiring service is reduced. Several problem instances are generated with a varying percentage of origins and destinations between which a load must be delivered. Each instance has 50, 100 or 150 origin-destination pairs and the percentages of these pairs requiring service is $100 \%, 80 \%, 60 \%, 40 \%$, and $20 \%$. Nine different sets of load sizes and three sets of location coordinates are randomly generated for each percentage, resulting in 27 problem instances for each number of loads requiring service. All load sizes are in the range $[0.51-0.60]$. Problem instances are generated for the ratios $5: 10,5: 20,10: 10$ and $10: 15$.

Figure 4 presents the cost reduction for the various instances. As the percentage of origin-destination pairs requiring service decreases, the cost reduction decreases as well. This can be attributed to a similar factor that caused the change in benefit as the ratio of origins to destinations approaches one. As the percentage of origin-destination pairs requiring service is reduced, each origin or destination has fewer loads to select from when creating a combination to place on a vehicle. This is most evident with the $5: 10$ ratio problem instances with $20 \%$ of pairs requiring service. Each origin has only one to three loads departing, while each destination has one load arriving. With fewer opportunities 

has limited benefit.

Fig. 4. Average Percentage Cost Reduction with Split Loads when the Percentage of Origin-Destination Pairs with a Load Requiring Service Varies

to split and combine loads onto a vehicle, the potential benefit is diminished. This is an indicator that in those real world situations with many isolated locations that have a limited number of loads requiring service, splitting loads

\section{Origin and Destination Location}

The effect of the location of the origins and destinations on the benefit of split loads has not been tested. Nowak et al. (2008) used locations uniformly generated over the test area for the random problem instances. In this section, several different location configurations that correspond to real world scenarios are tested.

One common scenario that occurs in the real world is that of origins clustered separately from destinations. In the auto industry, parts suppliers are closely located while production facilities are also clustered together. There is not much movement within these two clusters, with most shipments moving between the clusters. To evaluate the change in benefit associated with clustering, several different problem instances are generated.

Location coordinates are generated in three different configurations, A, B and C. For Configuration A, the X and Y coordinates for the origin are both randomly generated in the range $(0,30)$ while the destination coordinates are generated in the range $(-30,0)$, such that the two clusters are separate but 

destination, further increasing the potential benefit.

Fig. 5. Average Percentage Cost Reduction with Split Loads when Origins and Destinations are Clustered

adjacent. The origin and destination clusters are spaced further apart in Configurations B and C, where they are separated by a minimum of 30 and 60 units, respectively. Six sets of location coordinates and five sets of load sizes are randomly generated for each configuration, resulting in 30 problem instances. All load sizes are in the range $[0.51-0.60]$. Problem instances are generated for the ratios $5: 10,5: 20,10: 10$ and $10: 15$.

Figure 5 presents the cost reduction for both the random and clustered problem instances. Clustered origins and destinations result in a significant increase in cost reduction over randomly located origins and destinations. Splitting loads leads to more trips from origin to origin or destination to destination and fewer trips from an origin to a destination, as the vehicle picks up smaller loads from several origins rather than picking up a large unsplit load from one origin that is immediately transported to its destination. Clustering origins and destinations separately increases the average distance between origins and destinations relative to the average distance between origins or between destinations. Because splitting loads reduces the number of trips from origins to destinations, clustering leads to an increase in the potential benefit from splitting. As seen with Configurations B and C, lengthening the distance between the clusters increases the average distance from an origin to a

Another scenario tested separates the locations into three geographically divided clusters. Each cluster consists of several origins and destinations. Loads are delivered primarily within each cluster, with a few loads delivered between 

18 problem instances. All load sizes are in the range $[0.51-0.60]$.

\section{Table 2}

Number of Origins $\times$ Number of Destinations for each Cluster within each Configuration

clusters. These problem instances are similar to a real world scenario in which loads are transported within several regions, with very few delivered between regions, such as with a retail distribution network.

Three configurations are tested, each with a different number of origins and destinations in the three clusters. These configurations are described in Table 2. Problem instances are also generated with different restrictions on the number of loads that required service between clusters: instances with no loads requiring service between clusters, instances with approximately $50 \%$ of all loads requiring service to be delivered between clusters, and instances with up to $85 \%$ of all loads that may be delivered between clusters. Six sets of location coordinates and three sets of load sizes are randomly generated for each configuration and level of allowable inter-cluster load movement, resulting in
Table 3 presents the average percentage reduction in cost for each configuration and level of allowable inter-cluster load movement. As the number of inter-cluster moves increases, so does the reduction in cost. Just as with the problem instances presented above, where the origins and destinations are clustered separately, this is a result of an increase in the average distance that must be traveled to deliver loads between origins and destinations. Without inter-cluster moves the delivery of all loads occurs within the limited boundaries of a cluster. The average distance from an origin to a destination is the same as the average distance between two origins or between two destinations. 

to the number of moves between origins or between destinations.

Table 3

Average Percentage Cost Reduction with Split Loads for each Configuration with 324

As more inter-cluster moves are made, the average distance traveled by the vehicle from an origin to a destination increases. Allowing split loads results in a decrease in the number of moves between origins and destinations relative Various Restrictions on Moves Between Clusters

This result further underlines the usefulness of split loads when loads must be delivered over longer distances. Although benefit was found for instances where loads were only transported within the clusters, the cost reduction was markedly greater when loads were also delivered over the longer distances between clusters.

Altering the number of origins relative to the number of destinations per cluster also had an effect. Configuration A, which had an equal number of origins and destinations for each cluster, showed the least amount of cost reduction. As with the results found in Section 4, this configuration afforded the least opportunity to generate multiple split load combinations at each origin. When the number of origins and number of destinations in a cluster were not equivalent, the cost reduction increased.

\section{Conclusions}

The benefit associated with split loads varies considerably with most problem characteristics including load size, number of loads, and the configuration of origins and destinations. By testing various problem instances, we have found 
three primary factors that affect the benefit:

(1) Although some benefit was found with almost any mean load size and variance, those loads larger than one half of vehicle capacity showed the most potential, even with greater variances. These loads can not be combined without splitting, while they are the easiest to combine on a vehicle with splitting.

(2) As the number of loads available at a common location for pickup or delivery increases, so does the potential benefit from split loads. This is due to the increase in potential load combinations to split at each stop. This was shown by changing the ratio of the number of origins relative to the number of destinations, where the benefit decreased as the ratio approached one, and by decreasing the percentage of origin-destination pairs with a load requiring service, where the benefit decreased with the percentage.

(3) Increasing the average distance from an origin to a destination relative to the distance from origin to origin and destination to destination has a positive effect on the benefit of split loads. Because splitting loads reduces the number of trips from origins to destinations, clustering leads to an increase in the potential benefit from splitting. Both clustered scenarios supported this result, where clustering origins separately from destinations increased the reduction in cost from split loads as the clusters were spaced farther apart, while limiting the number of loads that could travel between the three separated clusters decreased the cost reduction. 


\section{References}

Archetti, C., Hertz, A., Speranza, M., 2006. A tabu search algorithm for the split delivery vehicle routing problem. Transportation Science 40, 64-73.

Archetti, C., Savelsbergh, M. W. P., Speranza, M., 2008. To split or not to split: That is the question, Transportation Research Part E. Forthcoming.

Dror, M., Laporte, G., Trudeau, P., 1994. Vehicle routing with split deliveries. Discrete Applied Mathematics 50, 239-254.

Dror, M., Trudeau, P., 1989. Savings by split delivery routing. Transportation Science 23, 141-145.

Dror, M., Trudeau, P., 1990. Split delivery routing. Naval Research Logistics $37,383-402$.

Frizzell, P. W., Giffin, J. W., 1995. The split delivery vehicle scheduling problem with time windows and grid network distance. Computers and Operations Research 22, 655-667.

Nowak, M. A., Ergun, O., White, III, C. C., 2008. Pickup and delivery with split loads, Transportation Science. Forthcoming.

Sierksma, G., Tijssen, G. A., 1998. Routing helicopters for crew exchanges on off-shore locations. Annals of Operations Research 76, 261-286. 